\title{
Direct numerical simulation of supersonic pipe flow at moderate Reynolds number
}

\author{
Davide Modesti ${ }^{*, \mathrm{a}, \mathrm{b}}$, Sergio Pirozzoli ${ }^{\mathrm{b}}$

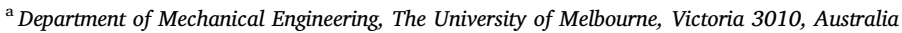 \\ ${ }^{\mathrm{b}}$ Dipartimento di Ingegneria Meccanica e Aerospaziale, Sapienza Università di Roma, via Eudossiana 18, Roma 00184, Italia
}

\section{A R T I C L E I N F O}

\section{Keywords:}

Pipe flow

Compressible flows

Wall turbulence

Direct numerical simulation

\begin{abstract}
A B S T R A C T
We study compressible turbulent flow in a circular pipe at computationally high Reynolds number. Classical related issues are addressed and discussed in light of the DNS data, including validity of compressibility transformations, velocity/temperature relations, passive scalar statistics, and size of turbulent eddies. Regarding velocity statistics, we find that Huang's transformation yields excellent universality of the scaled Reynolds stresses distributions, whereas the transformation proposed by Trettel and Larsson (2016) yields better representation of the effects of strong variation of density and viscosity occurring in the buffer layer on the mean velocity distribution. A clear logarithmic layer is recovered in terms of transformed velocity and wall distance coordinates at the higher Reynolds number under scrutiny $\left(R e_{\tau} \approx 1000\right)$, whereas the core part of the flow is found to be characterized by a universal parabolic velocity profile. Based on formal similarity between the streamwise velocity and the passive scalar transport equations, we further propose an extension of the above compressibility transformations to also achieve universality of passive scalar statistics. Analysis of the velocity/ temperature relationship provides evidence for quadratic dependence which is very well approximated by the thermal analogy proposed by Zhang et al. (2014). The azimuthal velocity and scalar spectra show an organization very similar to canonical incompressible flow, with a bump-shaped distribution across the flow scales, whose peak increases with the wall distance. We find that the size growth effect is well accounted for through an effective length scale accounting for the local friction velocity and for the local mean shear.
\end{abstract}

\section{Introduction}

Turbulent flow in circular pipes is common in many engineering applications, which cover both the incompressible and the compressible regime. Typical incompressible applications include transport of oil, potable, waste or irrigation water, whereas compressible pipe flow serves as prototype for the design of air intakes used in the aircraft industry. Several studies of incompressible pipe flow are available in the literature, which have highlighted similarities and differences with flow in planar channels. Eggels et al. (1994) carried out experiments and direct numerical simulation (DNS) of incompressible pipe flow at $R e_{b}=2 u_{b} R / \bar{\nu}_{w}=5300$ (where $u_{b}$ is the bulk flow velocity, $R$ is the pipe radius and $\bar{\nu}_{w}$ is the kinematic viscosity at the wall, respectively), and, in agreement with early experiments at low Reynolds number (Patel and Head, 1969), found that the mean velocity profile deviates from plane channel flow in the logarithmic and wake regions. Orlandi and Fatica (1997) carried out DNS of rotating and non-rotating pipe flow, and found good agreement with the experiments and numerical simulations by Eggels et al. (1994), in the non-rotating case. More recently, higher Reynolds numbers have been reached both in DNS (Wu et al., 2012; Chin et al., 2015; Lee et al., 2015; Ahn et al., 2015), up to friction Reynolds number $R e_{\tau}=u_{\tau} R / \bar{\nu}_{w} \approx 3000$ (where $u_{\tau}=\sqrt{\tau_{w} / \bar{\rho}_{w}}$ is the friction velocity, $\tau_{w}$ the mean wall shear stress and $\bar{\rho}_{w}$ the mean density at the wall), and experiments (Zagarola and Smits, 1998; Kim and Adrian, 1999; Mckeon et al., 2004; Furuichi et al., 2015), which are typically in the Reynolds number range $R e_{\tau} \sim 10^{3}-10^{5}$. These studies generally agree that the near-wall region of turbulent pipe flow is the same as in plane channel flow, whereas differences are found in the logarithmic and core regions. In particular, Kim and Adrian (1999) carried out experiments of pipe flow and observed that the pre-multiplied streamwise velocity spectra in the logarithmic layer are characterized by a peak at wavelengths corresponding to 24-28R, and referred to these structures as very-large-scale motions (VLSM). Wu et al. (2012) carried out DNS of incompressible pipe flow at friction Reynolds number $R e_{\tau} \approx 700$, and found a smaller spectral peak than in experiments, attributing the disagreement to use of

\footnotetext{
* Corresponding author at: Department of Mechanical Engineering, The University of Melbourne, Parkville, Melbourne, Victoria 3010, Australia.

E-mail address: davide.modesti@unimelb.edu.au (D. Modesti).
} 
Taylor's hypothesis (Alamo and Jiménez, 2009). Lee et al. (2015) and Ahn et al. (2015) showed that the population density of VLSM is lower in pipe than in channel flow, and that they survive for shorter time in pipes owing to wall confinement, which is the likely explanation for differences of the mean velocity profile in the outer layer.

Compressible pipe flow has received comparatively much less attention than the incompressible counterpart. Kjellström and Hedberg (1968) carried out experiments of compressible pipe flow at bulk Mach number $M_{b}=u_{b} / c_{w}=0.1-0.3$ (based on the wall speed of sound, $c_{w}$ ). At $M_{b}=0.3$ they noted compressibility effects on the wall shear stress, and claimed that accounting for compressibility corrections might be necessary for accurate measurements, even at low Mach number. Sandberg et al. (2012) carried out DNS of a fully developed compressible pipe flow exiting in a co-flow, and found good agreement of the pipe flow statistics with incompressible data up to a jet Mach number of 0.84. Ghosh et al. $(2006,2010)$ carried out the first DNS of supersonic turbulent flow in circular pipe and planar channel, and compared the flow statistics of the two geometries at matching Mach number and friction Reynolds number $\left(M_{b}=1.3, R e_{\tau}=245\right)$, and found that, as in incompressible flow, the mean flow statistics are affected by wall curvature effects.

The effect of fluid compressibility on mean velocity and Reynolds stresses is a long-standing issue in wall turbulence. Morkovin (1962) first postulated that genuine compressibility effects should be negligible if density fluctuations are smaller than mean density variations $\left(\rho^{\prime}<<\bar{\rho}\right)$, which became known as "Morkovin's hypothesis". This hypothesis led to several compressibility transformations for velocity and Reynolds stresses aimed at mapping compressible flow statistics onto equivalent incompressible ones through the mean density and viscosity profiles (van Driest, 1951; Coleman et al., 1995; Trettel and Larsson, 2016). In their DNS study of pipe flow, Ghosh et al. (2010) pointed out that the classical van Driest transformation (van Driest, 1951) is rather inaccurate, whereas good universality of the density-scaled Reynolds stresses was obtained using the semi-local scaling introduced by Huang et al. (1995). An extensive assessment of existing compressibility transformations was carried out by the present authors using DNS data of plane channel flow (Modesti and Pirozzoli, 2016). The results supported effectiveness of Huang's transformation in achieving universality of the Reynolds stress distributions. However, better universality of the mean velocity profile, with respect to classical van Driest transformation (van Driest, 1951), was obtained using the transformation proposed by Trettel and Larsson (2016) (hereafter referred to as TL transformation), based on the enforcement invariance of the logarithmic law in the overlap layer. The effect of compressibility on the velocity spectra, and therefore on the size of the turbulent eddies populating the wall layer, has also been studied for long time, with contradictory conclusions (Spina et al., 1994; Smits and Dussauge, 1996; Pirozzoli, 2011). Recent channel flow DNS (Modesti and Pirozzoli, 2016) and boundary layer experiments (Williams et al., 2018) have indeed shown that genuine compressibility effects on the typical flow scales are small, if any, and may be conveniently accounted for through variable-property extension of incompressible scaling formulas. Another subject of great interest in compressible flows is the transport of passive scalars, an essential building block in the understanding of mixing processes and combustion. Passive scalars in compressible wall-bounded flows have received little attention so far, mainly limited to the case of planar channels (Foysi and Friedrich, 2005). As in incompressible flow, similarity between passive scalars and the streamwise velocity field is also generally taken for granted in compressible flow, based on similarity of the governing equations.

In this work we develop a DNS database for compressible flow in a circular pipe which greatly extends the Reynolds number envelope of previous numerical studies. Our goal is to shed light on several facets of this flow which in our opinion have not been adequately addressed, so far. First, we aim at verifying the predictive power of compressibility transformations for mean velocity and Reynolds stresses, by also comparing with available incompressible DNS data at matching Reynolds number. Second, we aim at establishing scaling laws for the streamwise velocity spectra in the outer part of the wall layer, to quantify compressibility effects on the turbulent eddies. Third, we study the statistics and spectra of scalar fields passively advected by the fluid, with special emphasis on identifying similarities and differences with respect to the velocity field, and tracing possible compressibility effects. For that purpose, we carry out DNS in the range of bulk Mach numbers $M_{b}=0.3-3$, and of bulk Reynolds numbers $R e_{b}=5000-30000$, corresponding to friction Reynolds numbers $R e_{\tau}=180-1000$.

\section{Methodology}

We solve the compressible Navier-Stokes equations for a perfect shock-free heat-conducting gas in cylindrical coordinates, augmented with the transport equation for a passive scalar $\phi$,

$$
\begin{aligned}
& \frac{\partial \mathbf{w}}{\partial t}+\frac{1}{r}\left(\frac{\partial \mathbf{f}_{x}}{\partial x}+\frac{\partial \mathbf{f}_{r}}{\partial r}+\frac{1}{r} \frac{\partial \mathbf{f}_{\theta}}{\partial \theta}\right)-\frac{\partial \mathbf{f}_{x}^{v}}{\partial x}-\frac{1}{r} \frac{\partial r \mathbf{f}_{r}^{v}}{\partial r}-\frac{1}{r} \frac{\partial \mathbf{f}_{\theta}^{v}}{\partial \theta}+\mathbf{S}_{\mathbf{e}}-\mathbf{S}_{\mathbf{v}}-\mathbf{F} \\
& \quad=0
\end{aligned}
$$

where $\mathbf{w}$ is the vector of the conservative variables, and $\mathbf{f}_{i}, \mathbf{f}_{i}^{v} i=1,2,3$ are the convective and viscous fluxes in the coordinate directions $(x, r$, $\theta$ ),

$\mathbf{w}=\left[\begin{array}{c}\rho \\ \rho u_{j} \\ \rho s \\ \rho \phi\end{array}\right], \quad \mathbf{f}_{i}=\left[\begin{array}{c}\mathcal{R} u_{i} \\ \mathcal{R} u_{i} u_{j}+\mathcal{P} \delta_{i j} \\ \mathcal{R} u_{i} s \\ \mathcal{R} u_{i} \phi\end{array}\right], \quad \mathbf{f}_{i}^{v}=\left[\begin{array}{c}0 \\ \sigma_{i j} \\ q_{i} / T \\ J_{i}\end{array}\right] j=1,2,3$,

where $\mathcal{R}=r \rho, \mathcal{P}=r p$, with $\rho$ the fluid density, $p$ the thermodynamic pressure, and $s=c_{v} \ln \left(p \rho^{-\gamma}\right)$ the entropy per unit mass, $\left(\gamma=c_{p} / c_{v}=1.4\right)$. In Eq. (2), $\mathbf{S}_{\mathbf{e}}$ and $\mathbf{S}_{\mathbf{v}}$ are the Eulerian and the viscous source terms, and $\mathbf{F}$ contains the driving terms needed to keep the mass and scalar flow rate constant in time

$\mathbf{S}_{e}=\frac{1}{r}\left[\begin{array}{c}0 \\ 0 \\ -\rho u_{\theta}^{2}-p \\ \rho u_{r} u_{\theta} \\ 0 \\ 0\end{array}\right], \quad \mathbf{S}_{v}=\left[\begin{array}{c}0 \\ 0 \\ -\sigma_{\theta \theta} / r \\ \sigma_{r \theta} / r \\ \sigma_{\ell m} S_{\ell m} / T-q_{\ell} q_{\ell} / \lambda T^{2} \\ 0\end{array}\right], \quad \mathbf{F}=\left[\begin{array}{c}0 \\ \Pi \\ 0 \\ 0 \\ 0 \\ \Phi\end{array}\right]$,

where $\sigma_{i j}=2 \mu\left(S_{i j}-\frac{1}{3} \Theta \delta_{i j}\right)$, is the viscous stress tensor, $S_{i j}$ is the strain rate tensor,

$$
\begin{aligned}
S_{x x} & =\frac{\partial u}{\partial x}, & S_{x r} & =\frac{1}{2}\left(\frac{\partial u_{r}}{\partial x}+\frac{\partial u}{\partial r}\right), \\
S_{x \theta} & =\frac{1}{2}\left(\frac{\partial u_{\theta}}{\partial x}+\frac{1}{r} \frac{\partial u}{\partial \theta}\right), & S_{r r} & =\frac{\partial u_{r}}{\partial r}, \\
S_{r \theta} & =\frac{1}{2}\left(\frac{\partial u_{\theta}}{\partial r}+\frac{1}{r} \frac{\partial u_{r}}{\partial \theta}-\frac{u_{\theta}}{r}\right), & S_{\theta \theta} & =\left(\frac{1}{r} \frac{\partial u_{\theta}}{\partial \theta}+\frac{u_{r}}{r}\right),
\end{aligned}
$$

$\Theta$ is the dilatation,

$\Theta=\frac{\partial u}{\partial x}+\frac{\partial}{\partial r}\left(r \frac{\partial u_{r}}{\partial r}\right)+\frac{1}{r} \frac{\partial u_{\theta}}{\partial \theta}$,

and $q_{j}, J_{i}$ are the heat flux and scalar diffusion fluxes, respectively,

$$
\begin{array}{lll}
q_{x}=\lambda \frac{\partial T}{\partial x}, & q_{r}=\lambda \frac{\partial T}{\partial r}, & q_{\theta}=\lambda \frac{1}{r} \frac{\partial T}{\partial \theta}, \\
J_{x}=\rho \alpha \frac{\partial \phi}{\partial x}, & J_{r}=\rho \alpha \frac{\partial \phi}{\partial r}, & J_{\theta}=\rho \alpha \frac{1}{r} \frac{\partial \phi}{\partial \theta} .
\end{array}
$$

The dependence of the viscosity coefficient on temperature is accounted for through Sutherland's law, the thermal conductivity is defined as $\lambda=c_{p} \mu / \operatorname{Pr}$, with Prandtl number $\operatorname{Pr}=0.71$, and the scalar diffusivity is expressed in non-dimensional form in terms of the Schmidt 
number, $S c=\alpha / \nu$. Hereafter, the velocity components in coordinate directions will be denoted as $u, u_{r}, u_{\theta}$, and the variable $y=R-r$ will be used to denote the distance from the wall.

The equations are numerically solved using a fourth-order co-located finite-difference solver which guarantees discrete preservation of total kinetic energy from convection in the inviscid limit. For that purpose, the convective terms in Eq. (1) are first expanded to quasiskew-symmetric form, and then discretized using standard central difference formulas (Pirozzoli, 2010; 2011). The viscous terms are expanded to Laplacian form and similarly discretized with central difference formulas. The use of the entropy equation in place of the total energy equation is instrumental to semi-implicit time advancement, needed to discard the severe acoustic time step limitation in the azimuthal and wall-normal directions. As explained in Modesti and Pirozzoli (2018), this is realized through definition of a partial flux Jacobian matrix whereby only the effects of acoustic wave propagation are retained. Implicit treatment of the viscous terms in the azimuthal direction is also implemented to remove the viscous time step limitation at the pipe axis. The convective time step limitation in the azimuthal direction must also be alleviated in practical pipe flow calculations. For that purpose, the azimuthal convective derivatives are evaluated at progressively coarsened $\theta$ resolution as the axis is approached in such a way that the effective resolution in physical space is retained (Bogey et al., 2011).

The governing equations are solved in a cylindrical domain with size $L_{x} \times R \times 2 \pi R$. Periodicity is imposed in the streamwise and azimuthal directions, and no-slip isothermal boundary conditions are imposed at the wall, where we also set $\phi=0$. Regarding the singularity at the symmetry axis, we follow Mohseni and Colonius (2000), and stagger the first collocation point at a distance $\Delta r / 2$ from the axis. Hence no special treatment of the axis is required, provided the flow variables in the ghost points are suitably defined accounting for symmetry/antisymmetry conditions. The velocity and the passive scalar fields are initialized with the incompressible laminar solution, with superposed perturbations synthesized through the digital filtering technique (Klein et al., 2003), with initially uniform density and temperature. The flow statistics averaged in the streamwise and azimuthal directions have been collected at equally spaced time intervals, and convergence of the flow statistics has been checked a-posteriori. In the following we will use both Reynolds $\left(\phi=\bar{\phi}+\phi^{\prime}\right)$ and Favre $\left(\phi=\widetilde{\phi}+\phi^{\prime \prime}, \widetilde{\phi}=\overline{\rho \phi} / \bar{\rho}\right)$ averages, where the overline symbol denotes averaging in the streamwise and spanwise directions and in time. Accordingly, the Reynolds stress components are denoted as $\tau_{i j}=\bar{\rho} \widetilde{u_{i}^{\prime \prime} u_{j}^{\prime \prime}}$. Quantities normalized in wall-units $u_{v}, \delta_{v}$ (where $\delta_{v}=\bar{\nu}_{w} / u_{\tau}$ is the viscous length scale) are denoted with the + superscript.

The solver was preliminarily validated for the case of supersonic laminar flow. Fig. 1 shows the velocity and temperature profiles compared with a reference solution of the axisymmetric Navier-Stokes equations obtained using a standard ODE solver. The mean velocity profile is nearly (but not exactly) parabolic, whereas the temperature in the bulk flow is higher than at the wall temperature, as a result of aerodynamic heating. Overall, excellent agreement with the reference profile is observed.

A list of the computed flow cases is reported in Table 1. Three DNS have been carried out at bulk Mach number $M_{b}=1.5$ by changing the Reynolds number up to $R e_{\tau} \approx 1000$, and one DNS has been carried out at $M_{b}=3, R e_{\tau}=500$. Two additional DNS have been carried out, one at subsonic conditions to match reference incompressible DNS data by Wu and Moin (2008), and one at $M_{b}=1.3$, to match the conditions considered by Ghosh et al. (2010). Fig. 2 shows a comparison of the mean velocity profile and of the Reynolds stresses distributions. Agreement of the P02 flow case with reference data is excellent, and no compressibility effects are found at this low Mach number, as expected. Agreement is also good in general terms with the compressible pipe DNS by Ghosh et al. (2010). Significant offset with respect to the standard wall law is observed in this case, mainly because of poor effectiveness of van Driest transformation in accounting for density and viscosity variations in the near-wall layer, as discussed later on. Some differences with respect to the reference data are found as regards the peak of $\tau_{11}$. This is likely an effect of grid resolution, as Ghosh et al. (2010) used $\Delta x^{+}=9.5, R^{+} \Delta \theta \approx 12$, jointly with upwindbiased discretization. Inaccurate prediction of the wall shear stress is also the likely cause for the slight shift between the two velocity profiles in panel (c). Indeed, for the same bulk Reynolds number we find $R e_{\tau}=235$, whereas Ghosh et al. (2010) report $R e_{\tau}=245$. Hence, lower logarithmic intercept is expected in our case.

\section{Results}

\subsection{Velocity statistics}

Several attempts have been made in the past to remove compressibility effects from statistics of wall-bounded flows, starting from the classical analysis of van Driest (1951). Mean momentum balance in turbulent pipe flow requires

$\bar{\mu} \frac{\mathrm{d} \tilde{u}}{\mathrm{~d} y}-\bar{\rho} \widetilde{u^{\prime \prime} v^{\prime \prime}}=\bar{\rho}_{w} u_{\tau}^{2}(1-\eta)$,

where $\eta=y / R$ is the outer-scaled wall-normal coordinate. Away from the wall, molecular viscosity is negligible, and further assuming $\eta<<1$, constancy of the turbulent stress follows, hence

$-\widetilde{u^{\prime \prime} v^{\prime \prime}} \approx\left(\frac{\bar{\rho}_{w}}{\bar{\rho}}\right) u_{\tau}^{2}$

which shows that 'compressible' stresses should be scaled by the local mean density to recover the incompressible behavior, hence

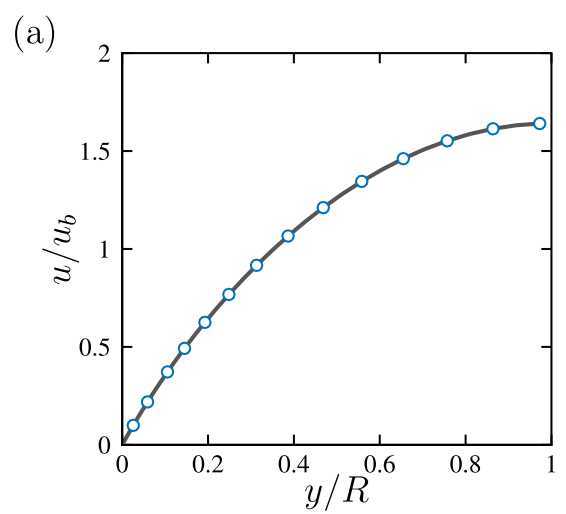

(b)

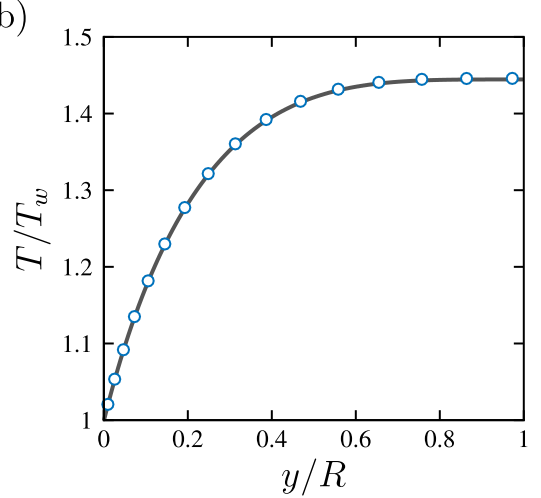

Fig. 1. Laminar supersonic flow in isothermal pipe at $M_{b}=u_{b} / c_{w}=1.5$ : radial profiles of velocity (a) and temperature (b). The DNS solution (circles) is compared with the reference one (solid lines), obtained using a standard ODE solver. 
Table 1

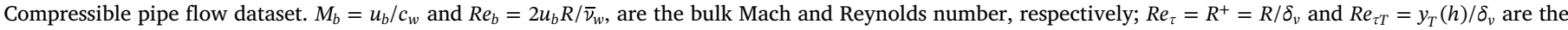

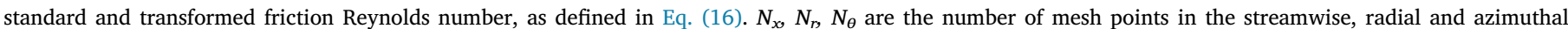

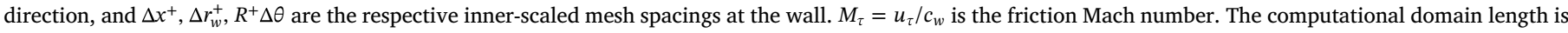
for all cases, with the exception of flow case P13 having $L_{x}=10 R$, for consistency with Ghosh et al. (2010). $\Delta t_{a v}$ is the time averaging interval.

\begin{tabular}{|c|c|c|c|c|c|c|c|c|c|c|c|c|}
\hline Case & $R e_{b}$ & $M_{b}$ & $R e_{\tau}$ & $R e_{\tau T}$ & $N_{x}$ & $N_{r}$ & $N_{\theta}$ & $\Delta x^{+}$ & $\Delta r_{w}^{+}$ & $R^{+} \Delta \theta$ & $M_{\tau}$ & $\Delta t_{a v} u_{\tau} / R$ \\
\hline P02 & 5300 & 0.2 & 184 & 180 & 256 & 64 & 256 & 11 & 4.5 & 0.014 & 0.0011 & 21.4 \\
\hline P13 & 6362 & 1.3 & 235 & 164 & 320 & 96 & 320 & 7.3 & 4.6 & 0.075 & 0.040 & 13.2 \\
\hline P15A & 6000 & 1.5 & 229 & 143 & 512 & 128 & 320 & 8.2 & 4.4 & 0.082 & 0.051 & 17.8 \\
\hline P15B & 14,600 & 1.5 & 521 & 334 & 1024 & 128 & 640 & 9.6 & 5.1 & 0.077 & 0.048 & 19.0 \\
\hline P15C & 31,500 & 1.5 & 1030 & 667 & 2048 & 256 & 1280 & 9.5 & 5.0 & 0.070 & 0.044 & 9.6 \\
\hline P3 & 10,300 & 3.0 & 524 & 147 & 1024 & 128 & 640 & 9.6 & 5.1 & 0.12 & 0.15 & 15.7 \\
\hline
\end{tabular}

(a)

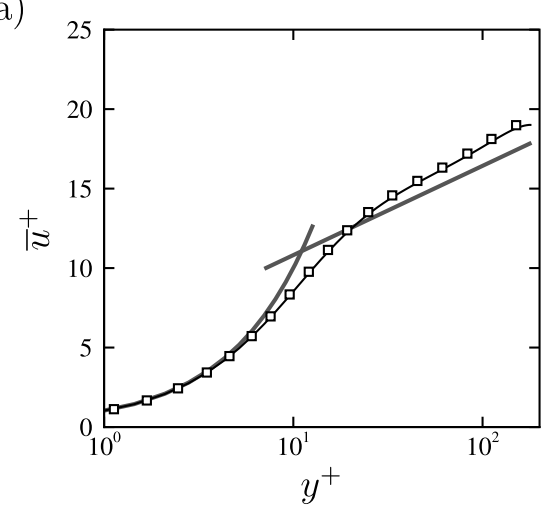

(c)

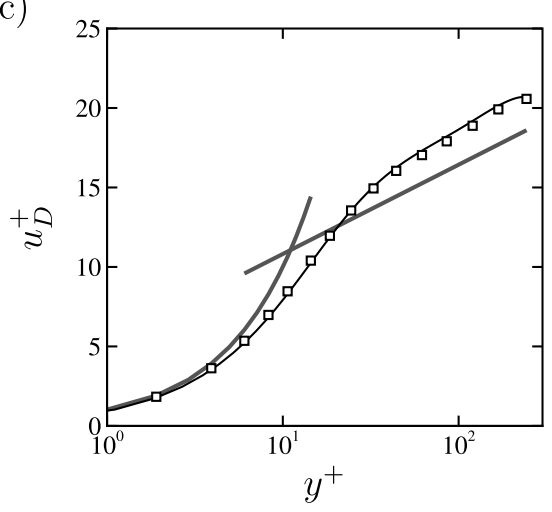

(b)

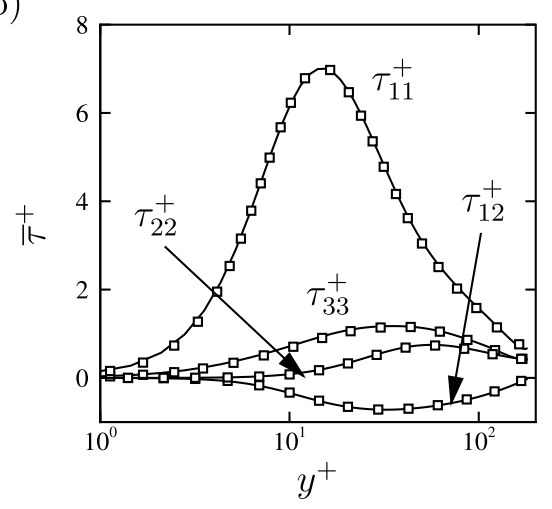

(d)

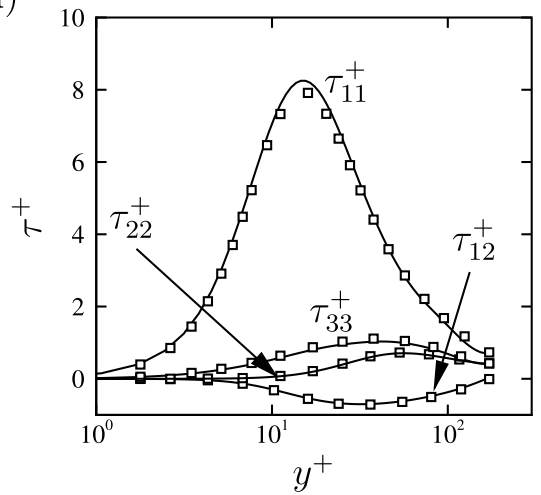

Fig. 2. Comparison of mean velocity profiles (left column) and turbulent stresses (right column) with reference literature data. (a), (b) comparison of P02 flow case with incompressible pipe DNS data by $\mathrm{Wu}$ and Moin (2008); (c), (d) comparison of P13 flow case with compressible pipe DNS data by Ghosh et al. (2010), where $u_{D}$ is the van Driest transformed velocity profile, defined in Eq. (11). Solid lines denote the present DNS results, and symbols the reference data. The thick gray lines in panels (a), (c) indicate the standard wall law, $\bar{u}^{+}=y^{+}, \bar{u}^{+}=1 / k \log \left(y^{+}\right)+B, k=0.41, B=5.2$. universality of the transformed stress tensor is expected

$\tau_{i j_{D}}=\frac{\bar{\rho}}{\bar{\rho}_{w}} \tau_{i j}$.

Mixing length modeling of the turbulent shear stress further leads to the classical overlap-layer equation

$\frac{d u_{D}}{d y}=\frac{u_{\tau}}{k y}$,

where $k$ is the von Kármán constant and $u_{D}$ the van Driest transformed velocity, defined as

$u_{D}=\int_{0}^{\bar{u}}\left(\frac{\bar{\rho}}{\bar{\rho}_{w}}\right)^{1 / 2} \mathrm{~d} \tilde{u}$.

Integration of (10) directly yields a logarithmic layer for the transformed velocity field with the same slope as in the incompressible case, however with an additive constant which may in general vary with both Reynolds and Mach number. It should be noted that Eq. (10) is obtained by neglecting viscous transport effects and mean viscosity variations which are dominant in the viscous sublayer, hence the van Driest transformation cannot be expected to yield universality in the entire wall layer. Since the error is mainly concentrated in the near-wall region, it may be expected that the van Driest transformation recovers its accuracy at sufficiently high Reynolds number, as the thickness of the viscous sublayer becomes negligible with respect to the wall layer thickness. It is also clear that in the case of adiabatic walls, since $\bar{\rho} / \bar{\rho}_{w} \approx 1$ in the near-wall region, van Driest transformation yields accurate results throughout the wall layer (Pirozzoli, 2011).

Failure of van Driest transformation was highlighted in previous studies, in which alternative transformations were proposed to map the whole wall layer. Empirical evidence (Huang et al., 1995) suggested scaling the wall-normal coordinate based on semi-local wall units, defined as

$u_{\tau}^{*}=\sqrt{\tau_{w} / \bar{\rho}}, \quad \delta_{v}^{*}=\bar{v} / u_{\tau}^{*}$,

yields better collapse of the flow statistics across the Mach number range. As it can be readily shown, this is equivalent to mapping the wall-normal coordinate according to

$y_{H}=\left(\frac{\rho_{w}}{\bar{\rho}}\right)^{1 / 2} \frac{\bar{\nu}_{w}}{\bar{v}} y$.

Trettel and Larsson (2016) further investigated failure of the van Driest 
(a)

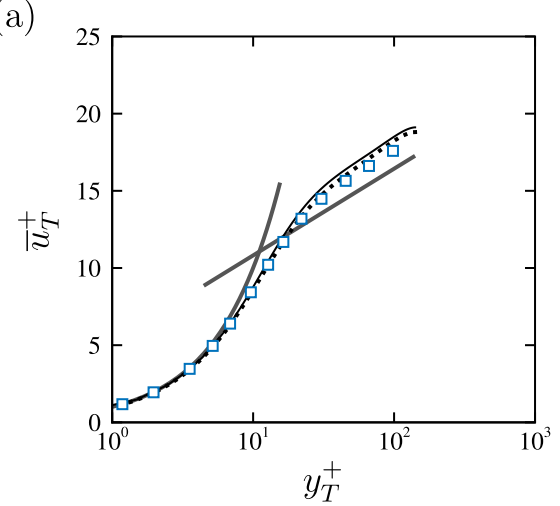

(c)

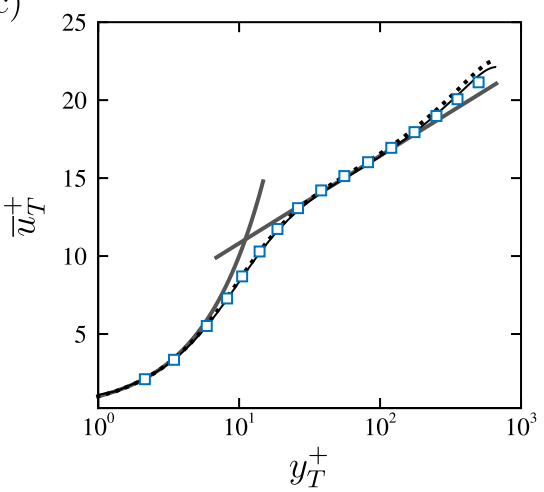

(b)

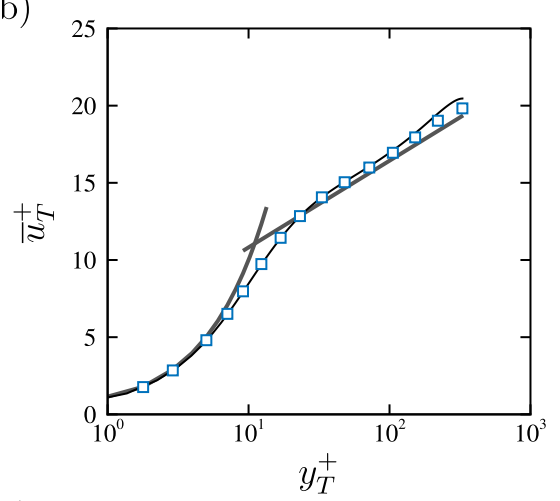

(d)

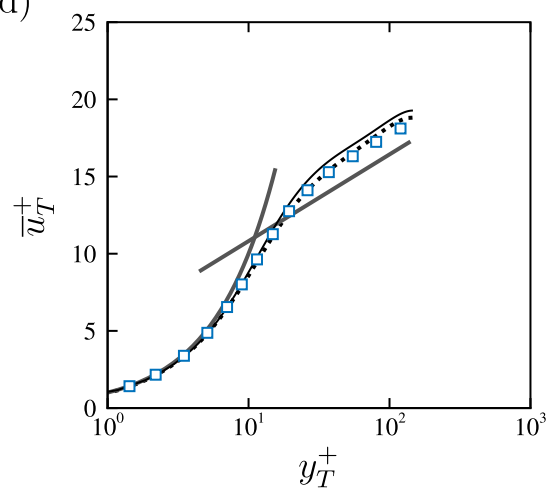

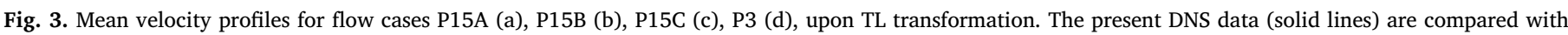

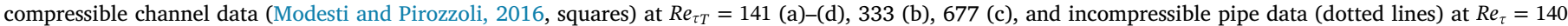

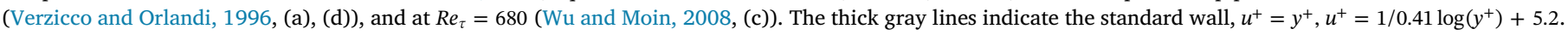

transformation in the case of cold walls, showing that Huang's semilocal scaling is actually rooted in arguments of mean momentum balance, and derived a novel velocity transformation which by construction satisfies universality of the turbulent stresses. The TL transformation can be expressed in terms of stretched space and velocity coordinates, defined as

$y_{T}(y)=\int_{0}^{y} f_{T}(\eta) \mathrm{d} \eta, \quad u_{T}(y)=\int_{0}^{y} g_{T}(\eta) \frac{d \tilde{u}}{d \eta} \mathrm{d} \eta$,

where

$f_{T}(y)=\frac{d}{d y}\left(\frac{y}{P^{1 / 2} N}\right), \quad g_{T}(y)=P N \frac{d}{d y}\left(\frac{y}{P^{1 / 2} N}\right)$,

with $N(y)=\bar{\nu} / \bar{\nu}_{w}, P(y)=\bar{\rho} / \bar{\rho}_{w}$. It is important to note that the viscousscaled wall normal coordinate defined in the first of Eq. (14) coincides with Huang's semi-local scaling given in Eq. (13). Fig. 3 shows the mean transformed velocity profiles obtained from DNS upon TL transformation, compared with reference compressible channel flow data (Modesti and Pirozzoli, 2016), and incompressible pipe flow data (Verzicco and Orlandi, 1996; Wu and Moin, 2008). For the sake of comparison, data are taken at similar values of the friction Reynolds number based on the transformed outer length scale, namely

$R e_{\tau T}=y_{T}(R) / \delta_{v}$

Fig. 3 shows general success of the TL transformation in mapping compressible DNS results back to incompressible ones across the Reynolds number range, with minor deviations at low $R e$, also observed in the case of plane channel flow at matching $R e_{\tau T}$. Instead, we find that van Driest transformation yields large deviations in the buffer layer which persist into the outer layer (the results are not shown, but one may refer to Fig. 2(c) for an indication). As in incompressible flows, large upward excursions from the standard log-law are found at low Reynolds number, whereas near logarithmic behavior is recovered starting from the P15C simulation. Comparison with channel flow data shows very similar distributions in the inner layer, with a slightly stronger wake region, again as in incompressible flow.

Similar conclusions can be drawn from scrutiny of the Reynolds stresses transformed according to Eq. (9), and shown in Fig. 4. All the Reynolds stress components actually collapse on the incompressible pipe distributions, with the exception of the buffer peak of $\tau_{11}$, whose offset however decreases with the Reynolds number. The peak overshoot of $\tau_{11}$ in compressible flow calculations is a well-known feature also in boundary layers (Pirozzoli and Bernardini, 2013) and channels (Modesti and Pirozzoli, 2016), and it is likely due to genuine compressibility effects, not fully explained so far. Close similarity with the stresses distributions in compressible channel is also observed, with differences again limited to the peak of $\tau_{11}$, and vanishing at higher Reynolds number. This is consistent with observations made regarding Reynolds number effects in the incompressible regime (Chin et al., 2014).

Closer insight into the core part of the flow can be gained by inspecting the mean velocity profiles in defect form, as given in Fig. 5. A parabolic law for the core velocity profile of incompressible pipe flow was derived by Pirozzoli (2014). The derivation stems from the idea that the outer-layer turbulent eddies are not directly affected by the presence of the wall, and their size should hence scale with the pipe radius and with the typical eddy velocity scale (namely the friction velocity), hence it follows that the relevant eddy viscosity is

$v_{t}=c_{\mu} u_{\tau} R$,

where $c_{\mu}$ is a suitable constant. This argument may be extended to compressible flow based on the assumption that in the presence of mean density variations the effective velocity scale is $u_{\tau}^{*}$ (as defined in Eq. (12)) rather than $u_{\tau}$, which yields the eddy viscosity

$v_{t}=c_{\mu} u_{\tau}^{*} R$ 
(a)

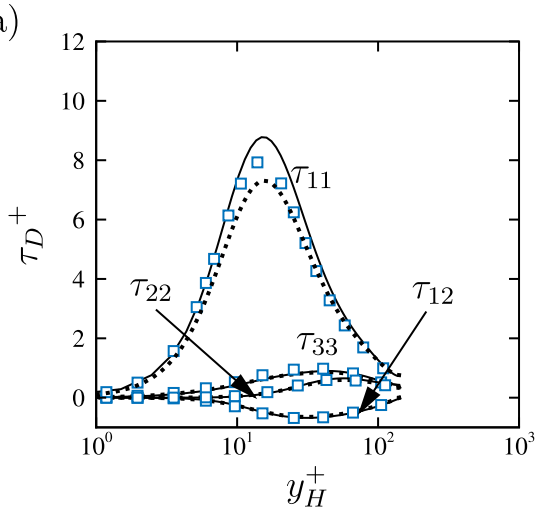

(c)

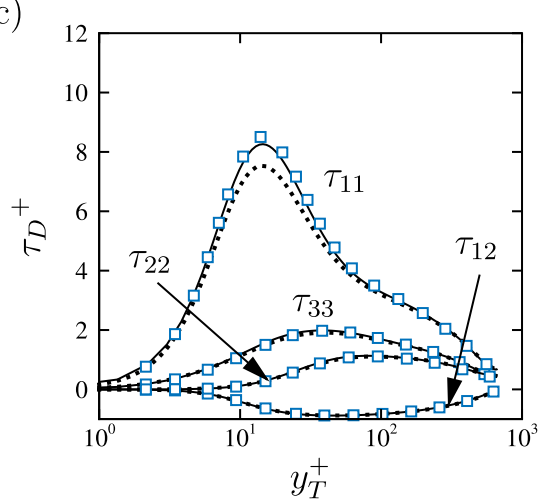

(b)

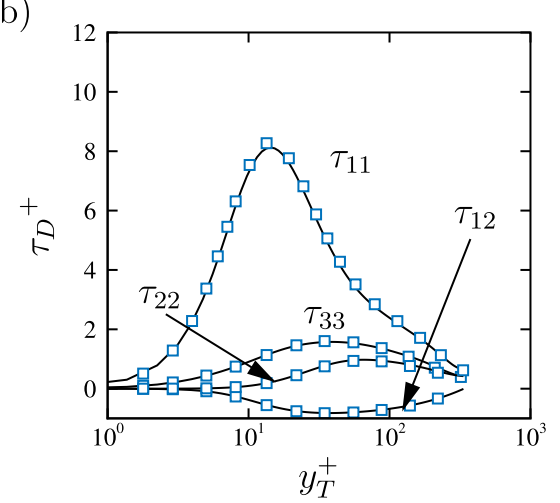

(d)

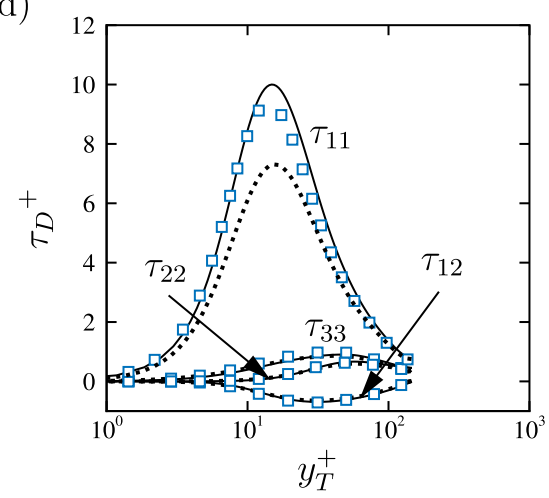

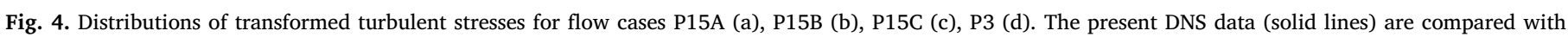

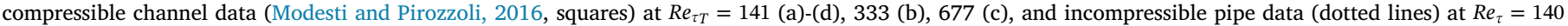
(Verzicco and Orlandi, 1996, (a), (d)), and at $R e_{\tau}=680$ (Wu and Moin, 2008, (c)).
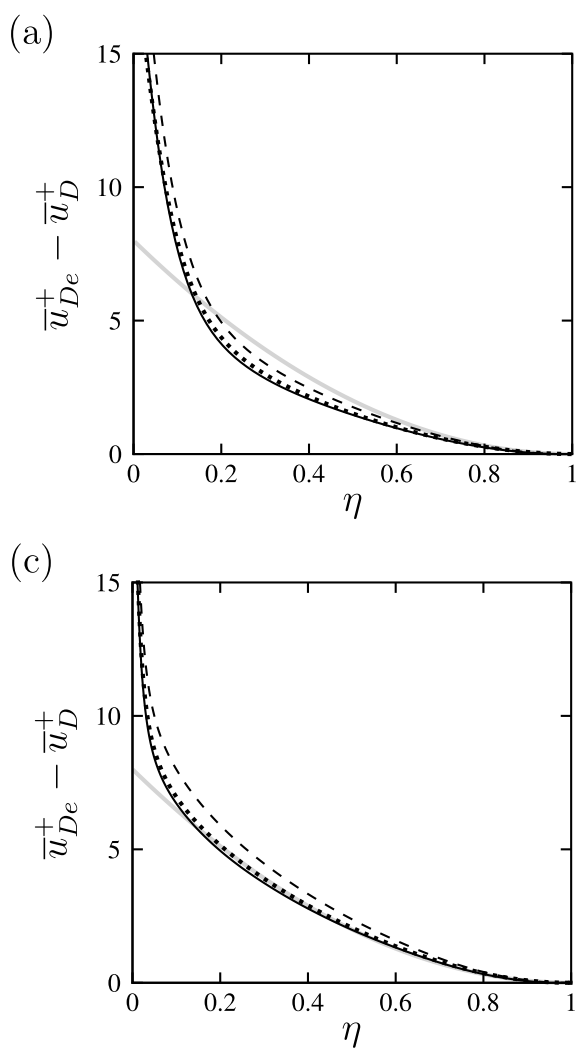

(b)

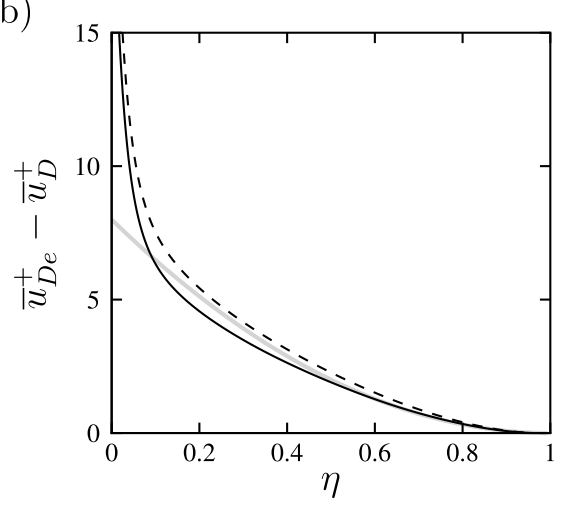

(d)

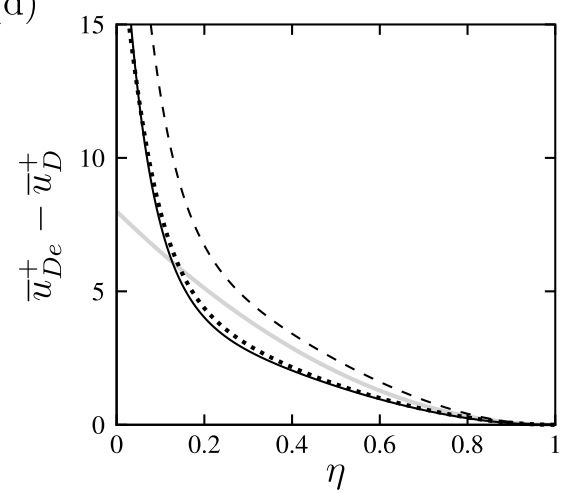

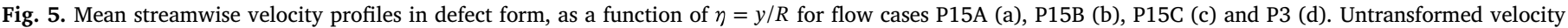

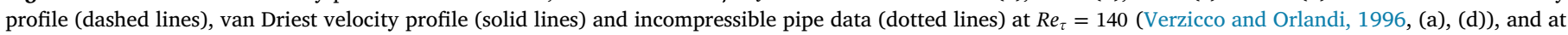

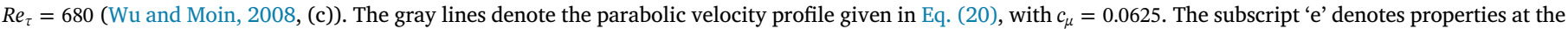
pipe centerline. 
From Eq. (7), neglecting the viscous term and using the eddy viscosity (18), one readily obtains

$\frac{\mathrm{d} \tilde{u}^{+}}{\mathrm{d} \eta}=\frac{1}{c_{\mu}}\left(\frac{\bar{\rho}_{w}}{\bar{\rho}}\right)^{1 / 2}(1-\eta)$,

from which it follows that the van-Driest-transformed velocity should follow a universal parabolic law in the core part of the pipe

$u_{D}^{+}-u_{D_{e}}^{+}=-\frac{1}{2 c_{\mu}}(1-\eta)^{2}$,

where $u_{D e}$ is the transformed centerline velocity. Outer defect profiles obtained with van Driest transformation are given in Fig. 5. Comparison with incompressible DNS (dotted lines) shows very good agreement throughout the outer layer, irrespective of the Reynolds and Mach number. The DNS data are consistent with the prediction of Eq. (20) around the pipe centerline, the range of validity of the parabolic fit extending to more than half pipe radius at sufficiently high Reynolds number. No evident compressibility effects are observed on the parabolic law constant, which in fact coincides with its incompressible value, namely $c_{\mu} \approx 0.0625$ (Pirozzoli, 2014). Similar results would be obtained with the TL transformation, as density and viscosity variations in the outer wall layer are but moderate.

\subsection{Temperature/velocity relationship}

An important subject in the study of compressible wall-bounded turbulence is that of temperature/velocity relations. Successful definition of a relation between temperature and velocity in fact allows to use inverse compressibility transformations (i.e. mapping incompressible velocity distributions to compressible ones), to derive explicit formulas for the friction coefficient (Smits and Dussauge, 1996). The classical temperature/velocity relation by Walz (1959) has proven its accuracy in the case of adiabatic walls (Duan et al., 2010), but it is found to fail in the case of isothermal walls (Modesti and Pirozzoli, 2016). Recently, Zhang et al. (2014) derived the following generalized temperature/ velocity relation,

$\frac{\widetilde{T}}{T_{w}}=1+\frac{T_{r g}-T_{w}}{T_{w}} \frac{\widetilde{u}}{\widetilde{u}_{e}}+\frac{\widetilde{T}_{e}-T_{r g}}{T_{w}}\left(\frac{\widetilde{u}}{\widetilde{u}_{e}}\right)^{2}$,

where $T_{r g}=\tilde{T}_{e}+r_{g} \tilde{u}_{e}^{2} /\left(2 c_{p}\right)$ is a generalized recovery temperature, $r_{g}=2 c_{p}\left(T_{w}-\tilde{T}_{e}\right) / \tilde{u}_{e}^{2}-2 \operatorname{Prq} q_{w} /\left(\tilde{u}_{e} \tau_{w}\right)$ is a generalized recovery factor, and $u_{e}$ and $T_{e}$ are the external values of velocity and temperature, here interpreted as the pipe centerline values. Eq. (21) explicitly takes into account the wall heat flux $q_{w}$, and it reduces to the Walz relation in the case of adiabatic walls. Fig. 6 shows mean temperature as a function of mean velocity for all points along the pipe radial direction. Nearly perfect superposition of the supersonic DNS data with the predictions of equation (21) is observed, especially at the highest $R e$. Integration of the inverse compressibility transformations (14) together with relationship (21) can then be exploited to reconstruct the mean velocity profile for assigned values of the bulk Reynolds and Mach number.

\subsection{Passive scalars}

The statistics of passive scalar fields at Schmidt number $S c=0.71$ and $S c=1$, are herein presented, with the main goal of comparing with the behavior of the streamwise velocity and temperature fields. The mean passive scalar balance equation reads

$\bar{\rho} \bar{\alpha} \frac{\mathrm{d} \tilde{\phi}}{\mathrm{d} y}-\bar{\rho} \widetilde{\phi^{\prime \prime} \nu^{\prime \prime}}=\bar{\rho}_{w} \phi_{\tau}^{2}(1-\eta)$,

where $\phi_{\tau}=\bar{\alpha}_{w} / u_{\tau}(\partial \widetilde{\phi} / \partial y)_{w}$ is the reference friction value. It is clear that for $S c=1$, Eq. (22)is identical to the mean momentum balance equation, Eq. (7), upon replacement of $\phi$ with $u$. This observation leads to expect that the TL transformation for velocity also applies to Eq. (22), thus allowing to map passive scalar distributions to their incompressible counterparts. Hence, by analogy with Eq. (14) we propose a transformation for $\phi$

$\phi_{T}(y)=\int_{0}^{y} g_{T}(\eta) \frac{d \tilde{\phi}}{d \eta} \mathrm{d} \eta$,

where $g_{T}$ is the same mapping function used for $u$, as defined in Eq. (15). Fig. 7 shows the inner-scaled transformed passive scalar profiles, compared with the correlations developed by Kader (1981) for incompressible pipe flow, which include a logarithmic layer with von Kármán constant $k_{\phi}=0.47$, and an additive constant varying with the Schmidt number. Good agreement is indeed recovered at sufficiently high Reynolds number, which is not surprising as Kader's correlations are based on data fitting of high-Reynolds-number experiments. This finding supports reliability of the TL transformation also for passive scalar fields. Comparison with the mean velocity profiles given in Fig. 3 shows similar behavior of mean velocity and mean passive scalar at $S c=1$, with the small but important difference that the log-law constant is $k \approx 0.41$ for $u$, and $k \approx 0.47$ for $\phi$. Fig. 7 (c) also reports passive scalar distributions in incompressible channel flow at matching $R e_{\tau T}$ (Pirozzoli et al., 2016). As for the velocity field, the agreement is excellent, with the exception of the wake region, where pipe flow exhibits a stronger wake component.

Temperature profiles in wall units are also shown in Fig. 7 to highlight differences with passive scalar profiles. It should be noted that temperature here is scaled with respect to its reference friction value, $T_{\tau}=\lambda_{w} /\left(\rho_{w} c_{p} u_{\tau}\right)(\partial \widetilde{T} / \partial y)_{w}$, where $\lambda_{w}$ is the fluid thermal conductivity at the wall. As expected, the inner-scaled temperature profiles well agree with the passive scalar profiles corresponding to $S c=0.71$ in the viscous sublayer, but they exhibit strong deviations thereof further away from the wall. Reasons for this difference may be explained by comparing the mean passive scalar and the mean temperature equations. The current flow case, with isothermal walls, shows perfect similarity of the equations with the exception of the forcing term, which is by construction spatially uniform in the equation for $\phi$, whereas the viscous dissipation term in the temperature equation is mainly concentrated in the vicinity of the wall.

Analogy of the mean momentum balance equation and the mean passive scalar equation further suggests that van Driest transformation may be used to map passive scalar variances and velocity/scalar correlations. From similarity with Eq. (9), we then consider the following mappings

$\left(\widetilde{\phi^{\prime \prime}}\right)_{D}=\frac{\bar{\rho}}{\bar{\rho}_{w}} \widetilde{\phi^{\prime \prime}}, \quad\left(\widetilde{\phi^{\prime \prime} u}\right)_{D}=\frac{\bar{\rho}}{\bar{\rho}_{w}}\left(\widetilde{\phi^{\prime \prime} u^{\prime \prime}}\right), \quad\left(\widetilde{\phi^{\prime \prime} v^{\prime \prime}}\right)_{D}=\frac{\bar{\rho}}{\bar{\rho}_{w}}\left(\widetilde{\phi^{\prime \prime} v^{\prime \prime}}\right)$.

Fig. 8 shows passive scalar fluctuations and scalar/velocity correlations for flow case P15C, transformed as in Eq. (24). The statistics are compared with passive scalar statistics in incompressible channel flow (Pirozzoli et al., 2016) at approximately matching $R e_{\tau T}$. Very good coincidence with the incompressible distributions is observed for the vertical scalar flux, see panel (c), whereas differences are observed for the near-peak of the scalar variance and of the streamwise scalar flux, which are consistently higher in the scaled compressible data. Again, this in similarity with the observations made regarding the buffer layer peak of the streamwise velocity variance when commenting Fig. 4. This is also an important clue that pressure, which is absent in passive scalar transport, does not play an important role in the breakdown of density scaling for the streamwise velocity variance peak, and reasons should be traced elsewhere.

As done for the mean velocity we now focus our attention on the mean passive scalar distributions in the core part of the pipe. Pirozzoli et al. (2016) showed that a universal parabolic profile holds for passive scalar fields in the core part of incompressible channels. The 
(a)

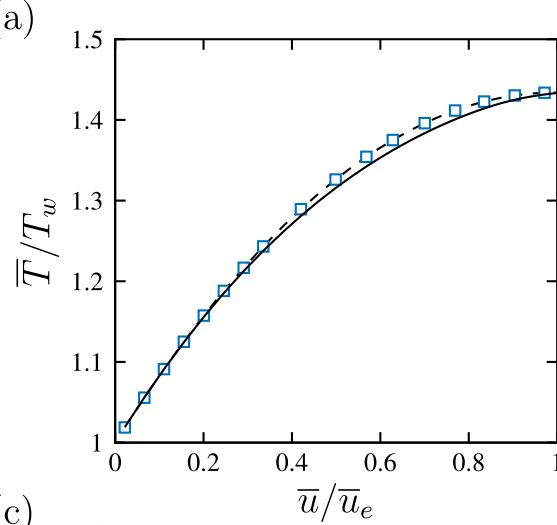

(c)

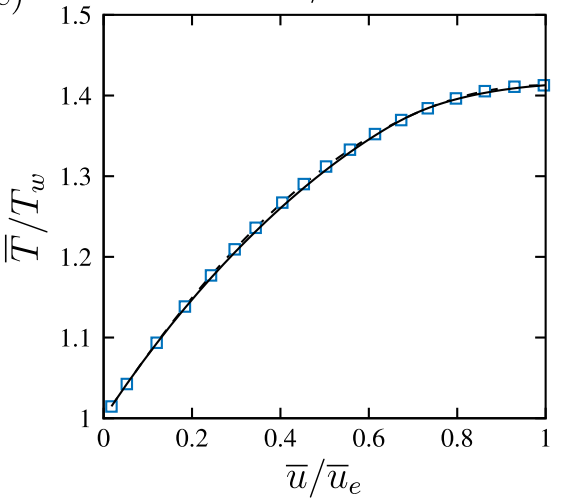

(b)

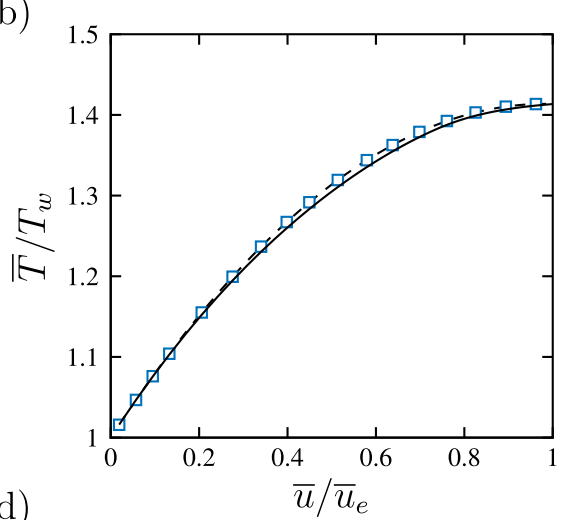

(d)

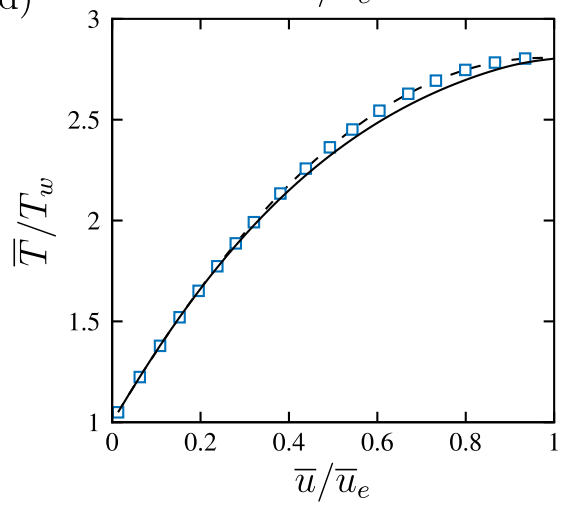

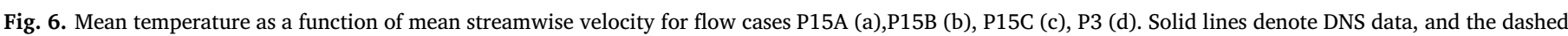
lines with squares indicate the predictions of Eq. (21).

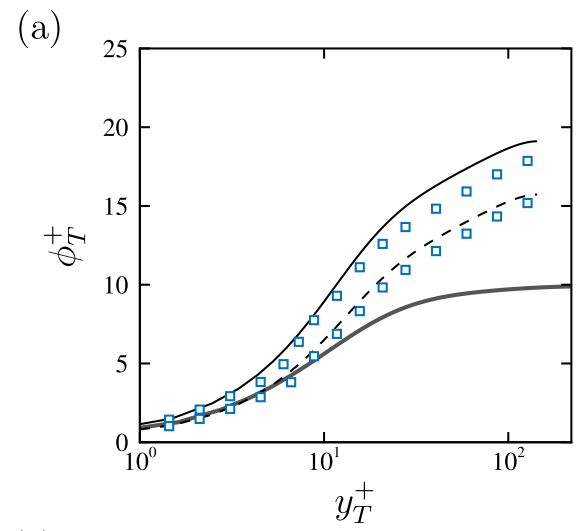

(b)
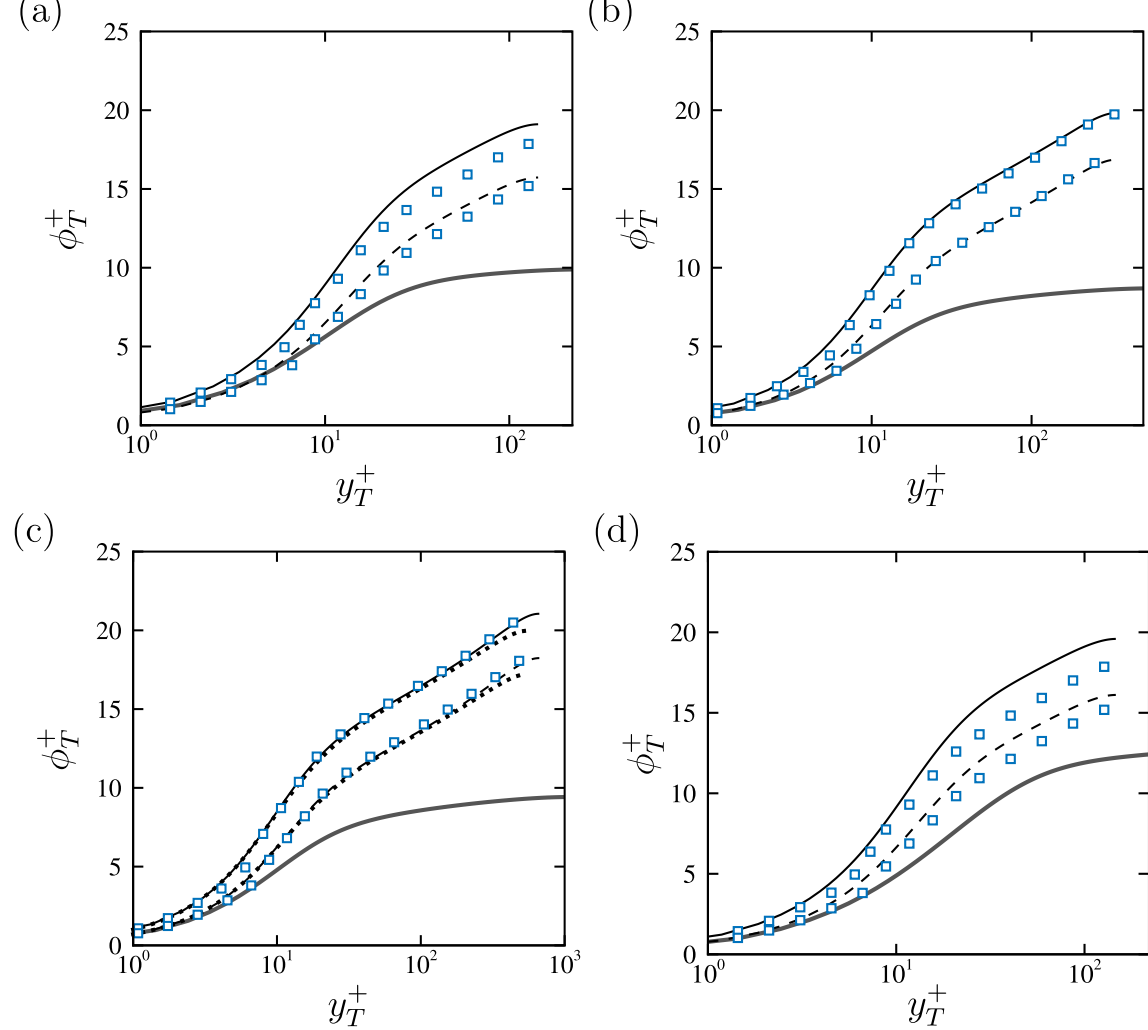

(d)

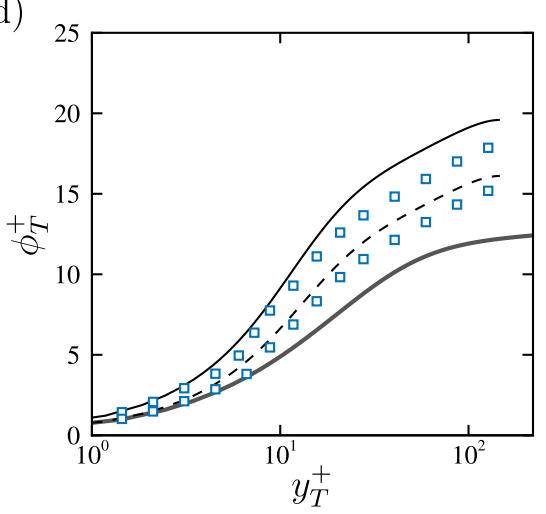

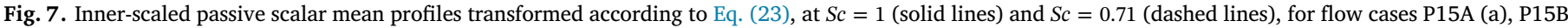

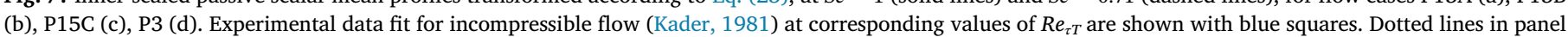

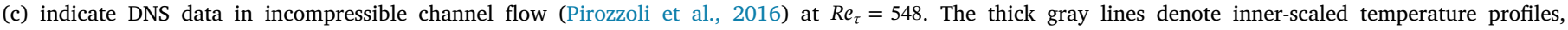
$T^{+}=\left(T-T_{w}\right) / T_{\tau}$. (For interpretation of the references to color in this figure legend, the reader is referred to the web version of this article.) 

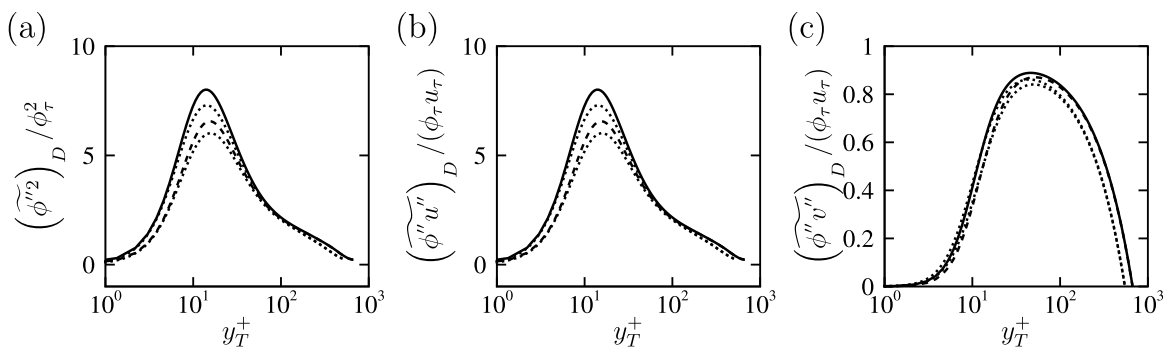

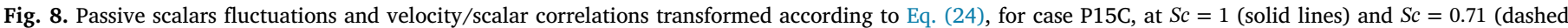
lines). The dotted lines denote the corresponding values in incompressible channel flow, at $R e_{\tau}=548$ (Pirozzoli et al., 2016 ).

same reasoning that led us to Eq. (20) can then be applied to the mean passive scalar balance equation (22), to obtain

$\phi_{D}^{+}-\phi_{D_{e}}^{+}=-\frac{1}{2 c_{\phi}}(1-\eta)^{2}$,

where

$\phi_{D}=\int_{0}^{\bar{\phi}}\left(\frac{\bar{\rho}}{\bar{\rho}_{w}}\right)^{1 / 2} \mathrm{~d} \tilde{\phi}$

is the mean passive scalar distribution transformed according to van Driest, and the subscript $e$ again denotes properties at the pipe axis. Outer defect profiles obtained with van Driest transformation are given in Fig. 9 at $S c=1,0.71$. The DNS data are very close to the prediction of Eq. (25)around the pipe centerline, the range of validity of the parabolic fit extending down to $\eta \approx 0.3$ at sufficiently high Reynolds number. No clear effects of compressibility and Schmidt number variation on the parabolic law constant are observed, and the latter very nearly coincides with the established incompressible value $c_{\phi} \approx 0.0693$ (Pirozzoli, 2014).

(a)

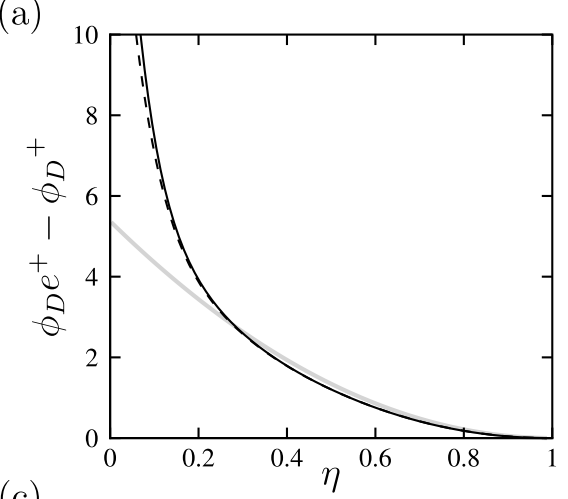

(c)

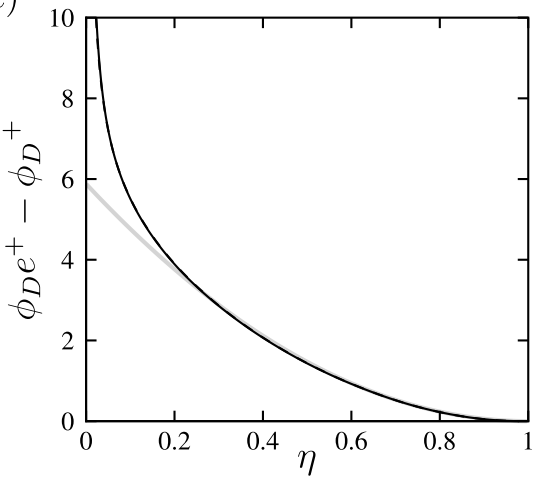

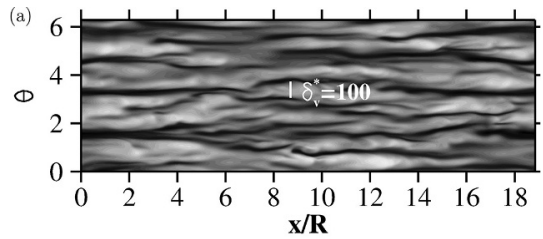

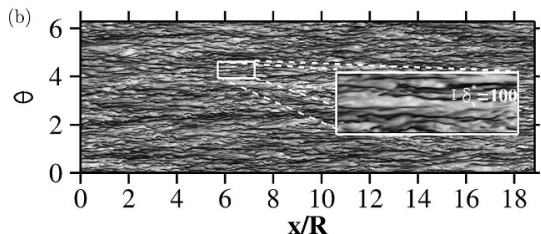

Fig. 10. Instantaneous streamwise velocity fluctuations for flow cases $P 15 A$ (a) and P15C (b) at $y_{T}^{+}=15$. Contours are shown in the range $-4 \leq u^{\prime \prime} / \sqrt{\tau_{11 D}} \leq 4$, from dark to light shades.

\subsection{Instantaneous flow field}

The general flow organization is scrutinized through visualization of the velocity and passive scalar fluctuations in the wall-parallel and cross-stream planes. Fig. 10 shows the instantaneous streamwise

(b)

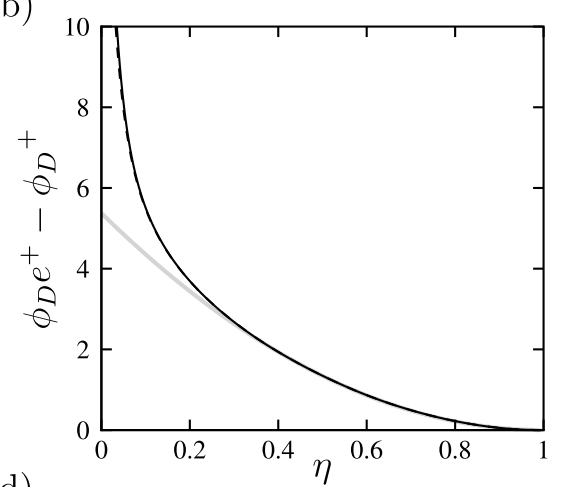

(d)

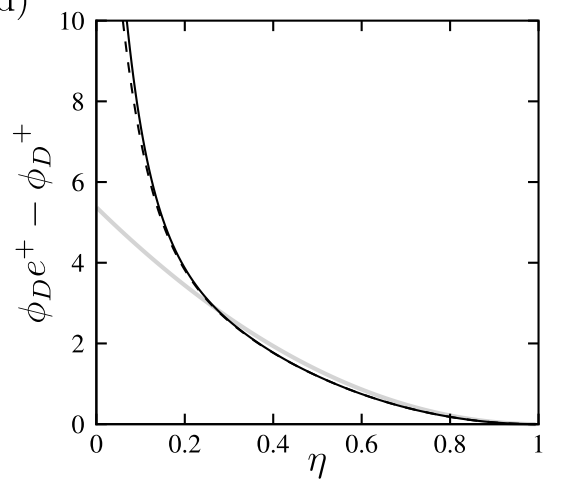

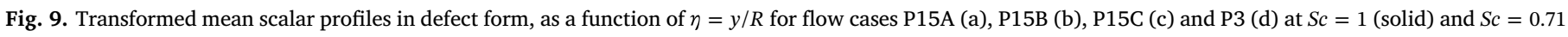
(dashed). The gray lines denote the parabolic velocity profile given in Eq. (25), with $c_{\phi}=0.0693$. The subscript 'e' denotes properties at the pipe centerline. 
(a)

(c)
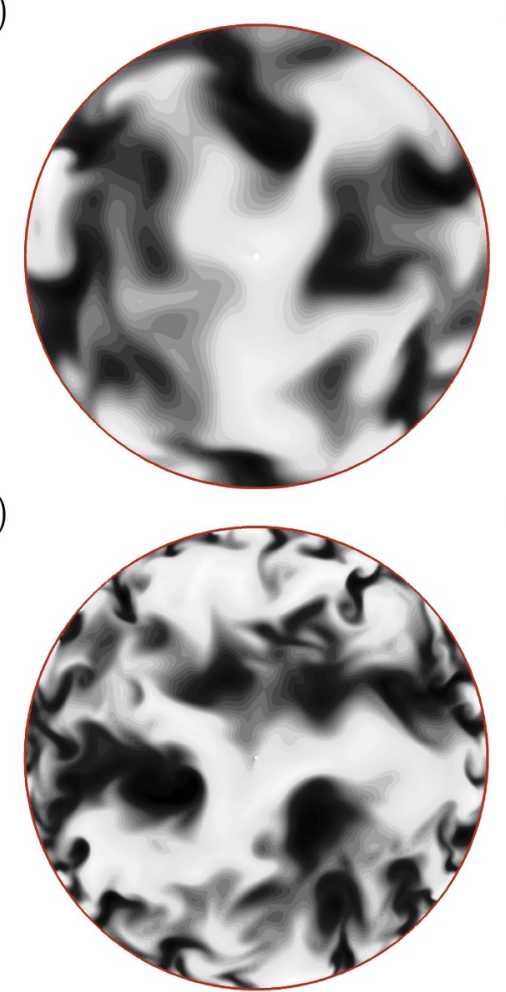

(e)

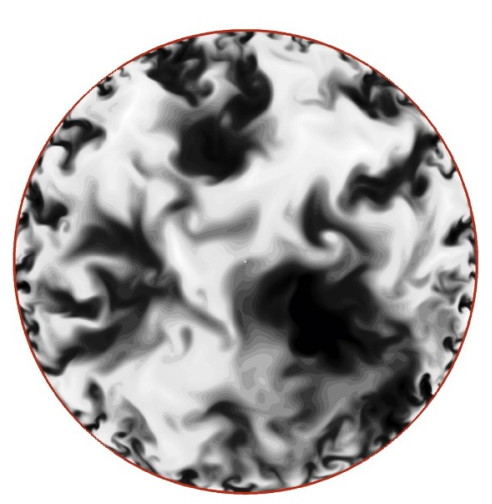

(g)

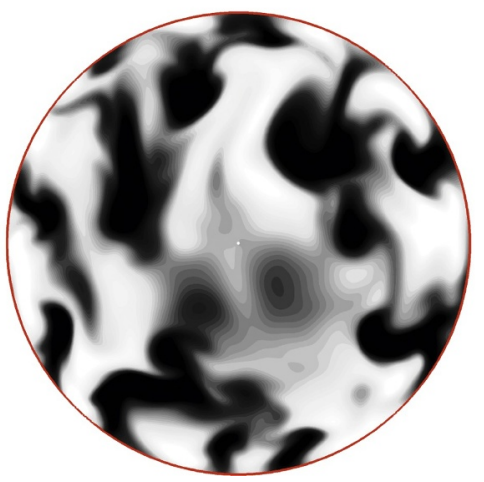

(b)

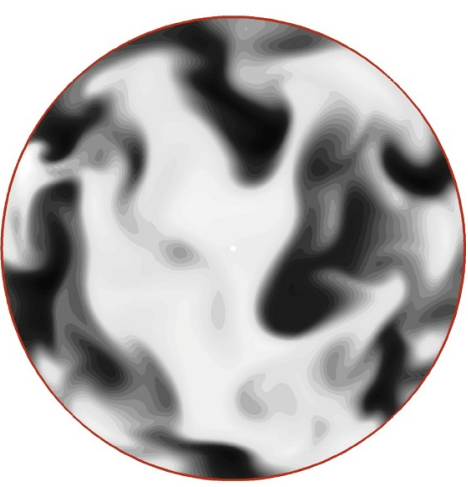

(d)

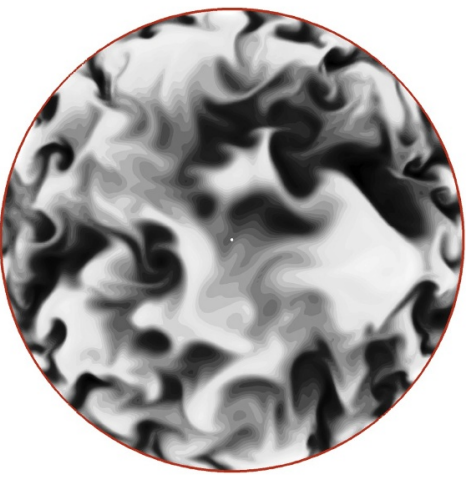

(f)

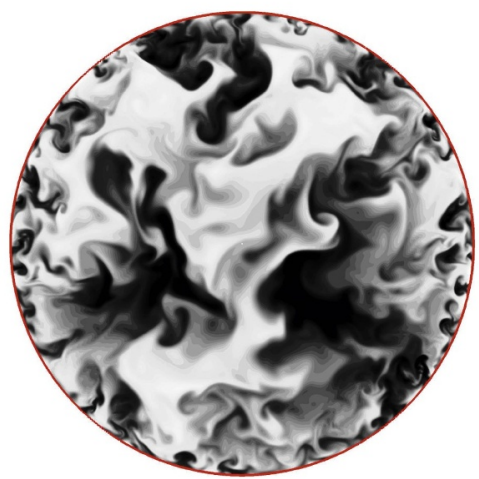

(h)

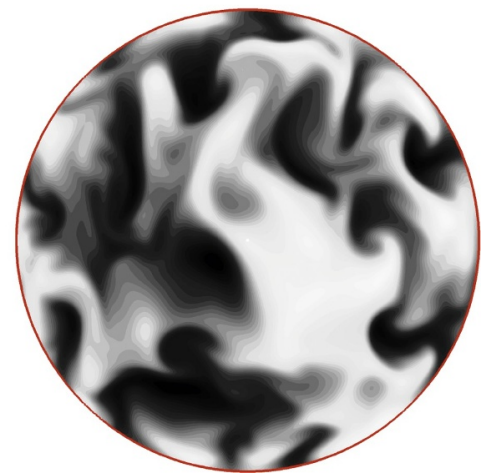

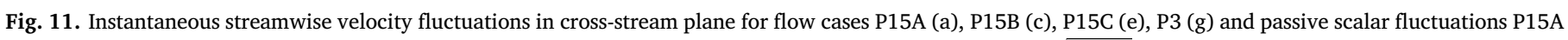

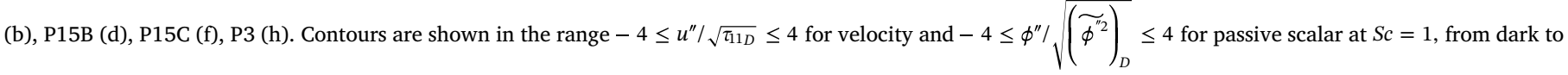
light shades.

velocity fluctuations on wall-parallel surfaces at $y_{T}^{+}=15$, for flow cases $\mathrm{P} 15 \mathrm{~A}$ and $\mathrm{P} 15 \mathrm{C}$. The velocity fluctuations in the buffer layer are organized in alternating low/high velocity streaky structures, elongated in the streamwise direction. The figure shows that the azimuthal spacing of the streaks decreases with the Reynolds number and in particular we find that the typical spacing is of order $100 \delta_{v}^{*}$, the same observed in incompressible flows, thus further supporting the use of the semi-local scaling (12) as effective wall-units in compressible flows. A 
complementary picture of the flow field may be gained by inspecting instantaneous flow snapshots in cross-stream planes, shown in Fig. 11. Whereas at low Reynolds number only eddies with $O(R)$ size are found, scale separation emerges at higher Reynolds number, with small-scale structures in the near-wall region and $O(R)$ eddies in the core region. As in incompressible wall-bounded flows, the passive scalar field shows substantial correlation with the streamwise velocity field, but it is characterized by sharper interfaces between regions with positive and negative fluctuations, owing to the absence of the pressure strain term which tends to smoothen the velocity field (Pirozzoli et al., 2016). The picture thus emerges that the qualitative structure of turbulence in compressible pipe flow is unaltered by compressibility, and the main features typical of all incompressible wall-bounded flows are retained.

\subsection{Length scales}

Based on the available DNS data we now turn to evaluating the typical length scales of turbulent eddies in the outer part of the wall layer. In a previous study of compressible channel flow (Modesti and Pirozzoli, 2016), no compressibility effect on the integral length scales was found. In that study we adapted to compressible flow the idea that in the outer layer the typical eddy length scale should depend on the friction velocity and the local mean shear (Pirozzoli, 2012). From dimensional analysis it directly follows, both for velocity and passive scalar, that in compressible flow the relevant length scales for streamwise velocity and passive scalar should be

$\ell_{12}^{*}(y) \sim\left(u_{\tau} R\right)^{1 / 2}\left(\frac{\mathrm{d} \tilde{u}_{D}}{\mathrm{~d} y}\right)^{-1 / 2}, \ell_{12 \phi}^{*}(y) \sim\left(\phi_{\tau} R\right)^{1 / 2}\left(\frac{\mathrm{d} \phi_{D}}{\mathrm{~d} y}\right)^{-1 / 2}$.

where $u_{D}$ and $\phi_{D}$ are defined in Eqs. (11)-(26), respectively. It should be noted that in the presence of a logarithmic layer, Eq. (27) would yield the classical scaling $\ell_{12}^{*}, \ell_{12 \phi}^{*} \propto y$ predicted by the attached eddy hypothesis. In order to evaluate the accuracy of the scaling given in Eq. (27), we consider the spanwise spectral densities of $u$ and $\phi$, defined such that

$\widetilde{u^{\prime \prime}}=\int_{0}^{\infty} E_{u}\left(k_{\theta}\right) d k_{\theta}, \quad \widetilde{\phi^{\prime \prime}}=\int_{0}^{\infty} E_{\phi}\left(k_{\theta}\right) d k_{\theta}$,

where $k_{\theta}$ is the Fourier wavenumber in the azimuthal direction. To eliminate effects due to variation of the turbulence intensity along the radial direction we actually consider the normalized spectral densities, defined as

$\hat{E}_{u}\left(k_{\ominus}\right)=E_{u}\left(k_{\theta}\right) / \widetilde{u^{\prime \prime}}, \quad \hat{E}_{\phi}\left(k_{\theta}\right)=E_{\phi}\left(k_{\theta}\right) / \widetilde{\phi^{\prime \prime}}$.

For the sake of the analysis, in the following we consider pre-multiplied, normalized energy spectra as a function of the azimuthal wavelength $\left(\lambda_{\theta}=2 \pi / k_{\theta}\right)$, in semi-log scale. This kind of representation provides hints about the distribution of energy across the flow scales with the illustrative advantage that equal areas underneath the graphs correspond to equal energies.

Fig. 12(a) and (b) shows pre-multiplied normalized velocity spectra at various wall distances across the wall layer, from $y^{+}=50$ to $\eta=0.7$. Standard outer scaling as a function of $\lambda_{\theta} / R$ is used in panel (a). It should be noted that that all spectra have a distinct bump shape, with energy concentrated at larger and larger scales moving away from the wall. In particular, in the outer layer, the energy peak corresponds to eddies with azimuthal size $\lambda_{\theta} \approx R$, closely resembling the spectral organization observed in incompressible flow (Kim and Adrian, 1999; Bailey and Smits, 2010), and consistent with the flow visualizations of Fig. 11. In panel (b) the same spectral densities are shown as a function of the scaled wavelengths $\lambda_{\theta} / \ell_{12}^{*}, \ell_{12 \phi}^{*}$. This type of representation does in fact yield significant improvement in the universality of the spectral densities, especially as far as the near-wall positions are concerned. The energy peak now is found to reside at $\lambda_{\theta}=2-3 \ell_{12}^{*}$, as also found in many incompressible flows (Pirozzoli, 2016). Based on the established similarity between streamwise velocity and passive scalars, we expect that the spectra of $u$ and $\phi$ are highly correlated. Indeed, Fig. 12(c) shows the same type of organization holds as for the streamwise velocity spectra, however the scalar spectra contain more energy at the smallest scales, owing to the absence of the pressure transfer term in the equation for $\phi$ (Pirozzoli, 2014), as also observed in Fig. 11. Similar to what found for the velocity field, we observe that the length scale $\ell_{12 \phi}^{*}$ yields improved collapse of the spectra across the wall layer (see panel (d)).

\section{Conclusions}

A DNS database for compressible turbulent flow in a straight circular pipe with isothermal walls has been developed, achieving Reynolds number significantly higher than previous studies, thus allowing to observe typical features of developed wall turbulence as a genuine logarithmic layer in the mean velocity distribution. The flow solver relies on a baseline central discretization of the convective derivatives expanded to quasi-skew-symmetric form, in such a way that discrete preservation of the total kinetic energy from convection is guaranteed. Efficient time stepping is guaranteed through semi-implicit treatment of acoustic waves in the convective terms, and local azimuthal coarsening near the pipe symmetry axis. Preliminary validation studies support good predictive capabilities of the solver.

Analysis of the velocity field generally supports findings of previous studies carried out in planar channel flow (Modesti and Pirozzoli, 2016). In particular, we find that Huang's transformation for the wall-normal coordinate coupled with standard density scaling yields near universality of the Reynolds stress components, the only exception being the peak streamwise velocity variance. Partial failure of Morkovin's hypothesis in this case is traditionally recognized as one of the few genuine compressibility effects, and sometimes traced to modifications of the pressure-strain term in the streamwise momentum equation (Foysi et al., 2004). However, we find that the same effect is also present in the passive scalar variance distributions also upon density scaling (see Fig. 8), in which the pressure term is not present. Hence, reasons for this (limited) lack of similarity may be even more subtle than previously thought. Van Driest transformation is found to be inaccurate in yielding universality of the mean velocity distributions, whereas the Trettel-Larsson transformation better accounts for mean density and viscosity variations taking place within the buffer layer. Excellent collapse with incompressible pipe data and compressible channel data is then achieved, and a sizeable canonical logarithmic layer is found to form at $R e_{\tau} \approx 1000$. The core part of the pipe is found to have a relatively simple structure, whereby the van Driest transformed velocity follows a universal parabolic law in a wide region, and it is controlled by a single universal constant, here found to be identical to that of incompressible pipe flow. As in other compressible wallbounded flows, the mean temperature distribution is found to quadratically depend on the mean streamwise velocity, and we find that the generalized Reynolds analogy of Zhang et al. (2014) yields very good prediction of its variation. Together with TL transformation, use of the temperature/velocity relation yields a closed system of equations, which in principle lends itself to closed formulas for the prediction of the friction and heat transfer coefficients, which might be the subject of further investigations.

The analysis of passive scalars in turbulent flow is interesting in its own sake, and because it allows to establish similarities/differences with respect to the streamwise velocity and temperature fields, which are governed by formally similar equations. Similarity with the velocity field are in fact strong, and we show that the TL transformation can be adapted to predict the mean incompressible passive scalar distributions. Temperature has on the other hand a very different behavior than a passive scalar, owing to strong spatial inhomogeneity of the viscous heating term. Finally, we have analyzed velocity and passive scalar spectra with the goal of establishing a parameterization for the growth 
(a)

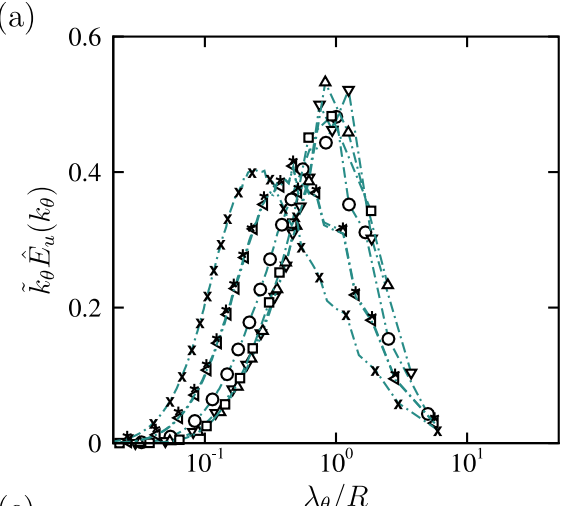

(c)

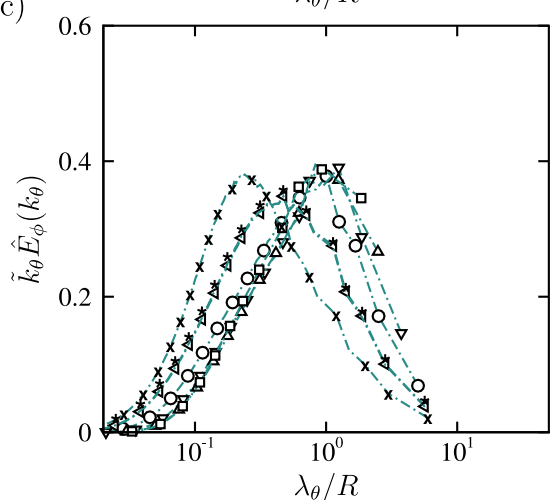

(b)

(d)
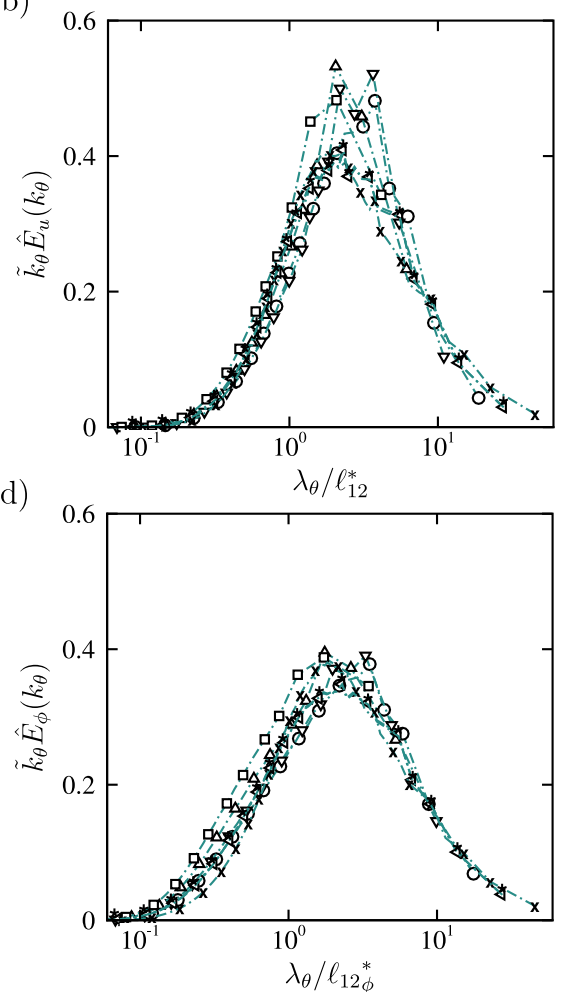

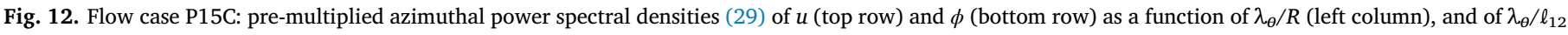

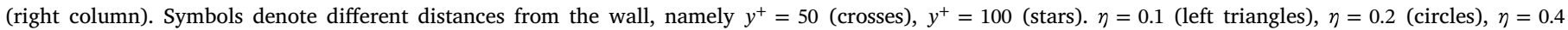
(gradients), $\eta=0.6$ (deltas), $\eta=0.7$ (squares).

of the typical eddy size along the wall-normal direction. In this respect we find that good universality of the spectral distributions is achieved when wavelengths are scaled with a length scale constructed with the local friction velocity defined in Eq. (12), and the local mean shear based on the van Driest transformed mean velocity profile. The same conclusion also applies to passive scalars. Flow statistics are available at the web page http://newton.dima.uniroma1.it/database.

\section{Acknowledgments}

We acknowledge that the results reported in this paper have been achieved using the PRACE Research Infrastructure resource MARCONI based at CINECA, Casalecchio di Reno, Italy.

\section{References}

Ahn, J., Lee, J., Lee, J., Kang, J., Sung, H., 2015. Direct numerical simulation of a 30R long turbulent pipe flow at $R e_{\tau}=3008$. Phys. Fluids (1994-present) 27 (6), 065110. Alamo, J.D., Jiménez, J., 2009. Estimation of turbulent convection velocities and corrections to Taylor's approximation. J. Fluid Mech. 640, 5-26.

Bailey, S., Smits, A., 2010. Experimental investigation of the structure of large-and verylarge-scale motions in turbulent pipe flow. J. Fluid Mech. 651, 339-356.

Bogey, C. Cacqueray, N.D., Bailly, C., 2011. Finite differences for coarse azimuthal discretization and for reduction of effective resolution near origin of cylindrical flow equations. J. Comput. Phys. 230 (4), 1134-1146.

Chin, C., Monty, J., Ooi, A., 2014. Reynolds number effects in DNS of pipe flow and comparison with channels and boundary layers. Int. J. Heat Fluid Flow 45, 33-40.

Chin, C., Ng, H., Blackburn, H., Monty, J., Ooi, A., 2015. Turbulent pipe flow at $R e_{\tau} \approx 1000$ : a comparison of wall-resolved large-eddy simulation, direct numerical simulation and hot-wire experiment. Comput. Fluids 122, 26-33.

Coleman, G., Kim, J., Moser, R., 1995. A numerical study of turbulent supersonic isothermal-wall channel flow. J. Fluid Mech. 305, 159-183.

van Driest, E., 1951. Turbulent boundary layer in compressible fluids. J. Aero. Sci. 18, 145-160.

Duan, L., Beekman, I., Martin, M., 2010. Direct numerical simulation of hypersonic turbulent boundary layers. Part 2: effect of wall temperature. J. Fluid Mech. 655, 419-445.

Eggels, J., Unger, F., Weiss, M., Westerweel, J., Adrian, R., Friedrich, R., Nieuwstadt, F.,
1994. Fully developed turbulent pipe flow: a comparison between direct numerical simulation and experiment. J. Fluid Mech. 268, 175-210.

Foysi, H., Friedrich, R., 2005. Passive scalar transport in turbulent supersonic channel flow. Progr. Turbulence. Springer, pp. 223-227.

Foysi, H., Sarkar, S., Friedrich, R., 2004. Compressibility effects and turbulence scalings in supersonic channel flow. J. Fluid Mech. 509, 207-216.

Furuichi, N., Terao, Y., Wada, Y., Tsuji, Y., 2015. Friction factor and mean velocity profile for pipe flow at high Reynolds numbers. Phys. Fluids (1994-present) 27 (9), 095108.

Ghosh, S., Foysi, H., Friedrich, R., 2010. Compressible turbulent channel and pipe flow: similarities and differences. J. Fluid Mech. 648, 155-181.

Ghosh, S., Sesterhenn, J., Friedrich, R., 2006. DNS and LES of compressible turbulent pipe flow with isothermal wall. Direct and Large-Eddy Simulation VI. Springer, pp. $721-728$.

Huang, P., Coleman, G., Bradshaw, P., 1995. Compressible turbulent channel flows: DNS results and modeling. J. Fluid Mech. 305, 185-218.

Kader, B., 1981. Temperature and concentration profiles in fully turbulent boundary layers. Int. J. Heat Mass Transf. 24 (9), 1541-1544.

Kim, K., Adrian, R., 1999. Very large-scale motion in the outer layer. Phys. Fluids 11 (2), 417-422.

Kjellström, B., Hedberg, S., 1968. Calibration Experiments with a DISA Hot-Wire Anemometer. Technical Report. AB Atomenergi.

Klein, M., Sadiki, A., Janicka, J., 2003. A digital filter based generation of inflow data for spatially developing direct numerical or large eddy simulations. J. Comput. Phys. 186 (2), 652-665.

Lee, J., Ahn, J., Sung, H., 2015. Comparison of large-and very-large-scale motions in turbulent pipe and channel flows. Phys. Fluids (1994-present) 27 (2), 025101.

Mckeon, B., Li, J., Jiang, W., Morrison, J., Smits, A., 2004. Further observations on the mean velocity distribution in fully developed pipe flow. J. Fluid Mech. 501, 135-147.

Modesti, D., Pirozzoli, S., 2016. Reynolds and Mach number effects in compressible turbulent channel flow. Int. J. Heat Fluid Flow 59, 33-49.

Modesti, D., Pirozzoli, S., 2018. An efficient semi-implicit solver for direct numerical simulation of compressible flows at all speeds. J. Sci. Comput. 75, 308-331.

Mohseni, K., Colonius, T., 2000. Numerical treatment of polar coordinate singularities. J. Comput. Phys. 157 (2), 787-795.

Morkovin, M., 1962. Effects of compressibility on turbulent flows. Mécanique de la Turbulence. A. Favre, pp. 367-380.

Orlandi, P., Fatica, M., 1997. Direct simulations of turbulent flow in a pipe rotating about its axis. J. Fluid Mech. 343, 43-72.

Patel, V., Head, M., 1969. Some observations on skin friction and velocity profiles in fully developed pipe and channel flows. J. Fluid Mech. 38 (1), 181-201.

Pirozzoli, S., 2010. Generalized conservative approximations of split convective derivative operators. J. Comput. Phys. 229 (19), 7180-7190.

Pirozzoli, S., 2011. Stabilized non-dissipative approximations of Euler equations in 
generalized curvilinear coordinates. J. Comput. Phys. 230 (8), 2997-3014.

Pirozzoli, S., 2012. On the size of the energy-containing eddies in the outer turbulent wall layer. J. Fluid Mech. 702, 521-532.

Pirozzoli, S., 2014. Revisiting the mixing-length hypothesis in the outer part of turbulent wall layers: mean flow and wall friction. J. Fluid Mech. 745, 378-397.

Pirozzoli, S., 2016. On the size of the eddies in the outer turbulent wall layer: evidence from velocity spectra. Progress in Wall Turbulence 2. Springer, pp. 3-15.

Pirozzoli, S., Bernardini, M., 2013. Probing high-Reynolds-number effects in numerical boundary layers. Phys. Fluids 25 (2), 021704.

Pirozzoli, S., Bernardini, M., Orlandi, P., 2016. Passive scalars in turbulent channel flow at high Reynolds number. J. Fluid Mech. 788, 614-639.

Sandberg, R., Sandham, N., Suponitsky, V., 2012. DNS of compressible pipe flow exiting into a coflow. Int. J. Heat Fluid Flow 35, 33-44.

Smits, A., Dussauge, J.-P., 1996. Turbulent Shear Layers in Supersonic Flow, Second ed. American Istitute of Physics.

Spina, E., Smits, A., Robinson, S., 1994. The physics of supersonic turbulent boundary layers. Annu. Rev. Fluid Mech. 26, 287-319.
Trettel, A., Larsson, J., 2016. Mean velocity scaling for compressible wall turbulence with heat transfer. Phys. Fluids (1994-present) 28 (2), 026102.

Verzicco, R., Orlandi, P., 1996. A finite-difference scheme for three-dimensional incompressible flows in cylindrical coordinates. J. Comput. Phys. 123 (2), 402-414.

Walz, A., 1959. Compressible turbulent boundary layers with heat transfer and pressure gradient in flow direction. J. Res. Natl. Bur. Stand. 63.

Williams, O., Sahoo, D., Baumgartner, M., Smits, A., 2018. Experiments on the structure and scaling of hypersonic turbulent boundary layers. J. Fluid Mech. 834, 237-270.

Wu, X., Baltzer, J., Adrian, R., 2012. Direct numerical simulation of a $30 R$ long turbulent pipe flow at $R e_{\tau}=685$ : large-and very large-scale motions. J. Fluid Mech. 698, 235-281.

Wu, X., Moin, P., 2008. A direct numerical simulation study on the mean velocity characteristics in turbulent pipe flow. J. Fluid Mech. 608, 81-112.

Zagarola, M., Smits, A., 1998. Mean-flow scaling of turbulent pipe flow. J. Fluid Mech. 373, 33-79.

Zhang, Y., Bi, W., Hussain, F., She, Z., 2014. A generalized Reynolds analogy for compressible wall-bounded turbulent flows. J. Fluid Mech. 739, 392-420. 